\title{
Vorstandsmitglieder und Chefmathematiker
}

$(V=$ Vorsitzender des Vorstandes; $0=$ ordentliches Vorstandsmitglied; st $=$ stellvertretendes Vorstandsmitglied)

1. Aachener und Münchener

Vorstand: Eugen Hirt (V), Konrad Seifferth (st).

Chefmathematiker: Rolf Schaffhirt.

2. Agrippina

Vorstand: Ernst Kahlo (o), Carl Bernheim (o) in Berlin;

Heinrich Paul Schriewer (st) in Kóln.

Chefmathematiker: Carl Bernheim.

3. Albingia

Vorstand: Hugo Weger (o), Johannes Woiff (0).

Chefmathematiker: Withelm Schramm.

4. Allgemeine Assekuranz

Vorstand: Gino Baroncini, Dr. Giuseppe Bufano, Dr. Alberto Melchiori.

Chefmathematiker: Dr. Vittorio Longo.

5. Allgemeine Rentenanstalt

Vorstand: Ludwig Luik (o), Dr. Wilhelm Paulus (o), Dr. Fritz Rueff (st).

Chefmathematiker: Dr. Fritz Rueff.

6. Allianz

Vorstand: Dr. Gerd Müller (V), Dr. h.c. Hans Parthier (0), Dr. Wolf Meister (o), Dr. Walter Mercker (o).

7. Alte Leipziger

Vorstand: Dr. Heinz Gehrhardt (o), Dr. Kurt Müller-Vollmer (o), Rudolf Schütz (o), Dr. Werner Zerbst (o).

8. Alte Volksfürsorge

Vorstand: Emil Thiele (V), Hans WeiBhaar (o), Fritz Börner (o), Gustav Dahrendorf (0), Dr. h. c. Henry Everling (0), Wilhelm Petersen (o), Heinrich Steinfeldt (o).

Chefmathematiker: Hermann Haack.

9. Atlas

Vorstand: Eugen Hirt (o).

Chefmathematiker: Rolf Schaffhirt.

10. Basler

Vorstand: Dr. Felix Vischer(V), Fürsprecher Theophil Thürkauf (o), Prof. Dr. Ernst Zwinggi (o), Dr. Jon Pinösch (o); Jakob Schaffner, Dr. Emilio Peduzzi, Ernst Suter, Subdirektoren.

Verantwortl. Mathematiker: Dr. Alexander Leepin.

Hauptbevollmächtigter für Deutschland: Fritz Simon in Frankfurt a. M. 
11. Bayerische Beamten

Vorstand: Georg Meier (V), Michael Schnappauf (o), Dr. Günther Wünsche (o).

Chefmathematiker: Dr. Günther Wünsche.

12. Berlinische

Vorstand: Dr. Arno Eberhard (V), Hans Reichold (o), Hans Kroll (0), Heinrich Först (st).

Chefmathematiker: Fritz Windrath.

13. Bonner

Vorstand: Hans Ludwig Stelges (V), Dr. Anneliesel Bertsche (o). Chefmathematiker: Dr. Anneliesel Bertsche.

14. Braunschweigische

Vorstand: Dr. Alexander Küchling (0), Dr. Willi Reiche (0). Chefmathematiker: Dr. Willi Reiche.

15. Concordia

Vorstand: Otto Garde (V), Hans Bodo Koebke (st), Dr. Hellmuth Nöbel (st), Fritz Heusing (st).

Chefmathematiker: Josef $M$. Seitz und Jakob Maller.

16. Debeka

Vorstand: Hermann Lange (V), Paul Jung (0), Matthias Gangolf(o), Dr. Clemens Schroeder (o).

Chefmathematiker: Dr. Clemens Schroeder.

17. Der Anker

Vorstand: Offentl. Verwalter Rudolf Karras.

Chefmathematiker: Rudolf Karras.

18. Deutsche Arzte

Vorstand: Walter Meewes (o), Berlin, Dr. Wilhelm Fricke (0), Hamburg.

Chefmathematiker: Walter Meewes.

19. Deutsche Beamten - Berlin

Vorstand: Carl Matschke (V), Berlin, Karl Blaß (o), München. Chefmathematiker: Dr. Gustav Kettler.

20. Deutsche Eisenbahn-Vers.-Kasse

Vorstand: Paul Beuche (V), Dr. Kurt Schaefer (stellv. V), Theodor Martens (o), Bernhard Meyer (o), Georg Mietz (o). Chefmathematiker: Carl Pürkner.

21. Deutscher Herold

Vorstand: Dr. Willy Günther (0), Wilhelm Hamann (0), Walter Hartung (o), Dr. Herbert Worch (0).

22. Deutscher Lloyd

Vorstand: Johannes Friedrich Schippmann (o), Erich Pallasch(st). Chefmathematiker: Dr. Albert Selow.

23. Deutscher Ring

Vorstand: Herbert Dau (V), Ehrich Reins (0), Max Raloff (0), Dr. Henry Mählmann (0).

Chefmathematiker: Dr. Henry Măhlmann.

24. Eos

Vorstand: Rudolf Baller (V), Erich A. Meyer (st).

Chefmathematiker: Erich A. Meyer. 
25. Friedrich Wilhelm

Vorstand: Dr. Hans Gerling (V), Walter Gerling (stellv. V), Rudolf Kratochwill (o), Dr. Wilhelm Taeffner (0).

Chefmathematiker: Dr. Wilhelm Taeffner.

26. Gerling-Konzern

Vorstand: Dr. Hans Gerling (V), Dr. Kurt Fischer (o), Peter Lenz (o), Edgar Prang (0).

Chefmathematiker: Dr. Kurt Fischer.

27. Gilde

Vorstand: Georg Stodt (V), Hermann Behrens (o), Elisabeth Lutz (o), Karl Schrödter (0).

Chefmathematiker: Karl Schrödter.

28. Gisela

Vorstand: Richard Stellwag (0), Albert Gift (st).

Chefmathematiker: Kriemhild Etzel.

29. Gladbacher

Vorstand: Dr. Gustav Harting (V), Dr. Paul Beck (0).

Chefmathematiker: Friedrich Henning.

30. Gothaer

Vorstand: Dr. Gerhard Frels (V), Dr. Herbert Haasen (0),

Oswald Heinig (o), Dr. Julius Winter (0), Alex Scherf(st).

Chefmathematiker: Dr. Walter Schwarzburg.

31. Hamburg-Mannheimer

Vorstand: Dr. Hermann Hitzler (V), Dr. Theodor Dörstling (o), Dr. Paul March (o).

Chefmathematiker: Dr. C. Raabe.

32. Hannoversche

Vorstand: Dr.Rudolf Kaewel (o), Johann Engelken (0), Dr. Werner Semmler (0).

Chefmathematiker: Karl Arndt.

33. Hansa

Vorstand: Dr. Hermann Hitzler (V), Dr. Theodor Dörstling (o), Dr. Paul March (o).

Chefmathematiker: Dr. C. Raabe.

34. Iduna-Germania

Vorstand: Berthold Beitz (V), Dr. Wilhelm Hartmann (0),

Dr. Heinrich Rütten (o), Rudolf Meisch (st), Gunther Albers (st).

Chefmathematiker: Rudolf Meisch.

35. Isar

Vorstand: Walter Giesel (0), Dr. Eckart Weinreich (0).

Chefmathematiker: Georg Stitzl.

36. Karlsruher

Vorstand: Dr. e. h. Alex Möller (V), Ernst Grevemeyer (0), Dr. Heinz Closs (0).

Chefmathematiker: Dr. Paula Schweiger.

37. Kölnische

Vorstand: Dr. Joseph Hecking(0), Dr. Josef Burauen (o), Emil Josef Kohlhaas (0).

Chefmathematiker: Dr. Josef Burauen.

38. Lebensversicherungsanstalt Berlin

Vorstand: Dr. Willy Müller-Wieland (V), Gerhard Loch (st).

Chefmathematiker: Erich Friede.

39. Leipziger Verein-Barmenia

Vorstand: Horst Kadatz (o), Wilhelm Schenkmann (st).

Chefmathematiker: Ilse Turley. 
40. Magdeburger Allgemeine

Vorstand: Dr. Rudolf Rebsamen (V), Dr. Karl Rudolph (0),

Dr. Claus Fischer (0).

Chefmathematiker: Dr. Ludwig Bentfeldt.

41. Magdeburger Leben

Vorstand: Dr. Hans Gerling (V), Walter Gerling (stellv. V), Dr. Edgar John (0), Dr. ing. Helmut Kracke (0).

Chefmathematiker: Dr.ing. Helmut Kracke.

42. Mannheimer

Vorstand: Martin Bothe (o), Arthur Braune (st).

Chefmathematiker: Hildegard Westhoff.

43. Münchener Leben

Vorstand: Dr. Alfred Brachvogel (0), Dr. Chr. Albert Franke (0).

Chefmathematiker: Karl Kirchhof.

44. Münchener Verein

Vorstand: Hans G. Lust (V), Dr. Hans Hieber (o).

Chefmathematiker: Dr. F. Stutz.

45. National

Vorstand: Dr. Werner Plath (V), Dr. Ernst Kircher (o), Dr. Erich Carus (0).

Chefmathematiker: Rudi Queiker.

46. Nord-Deutsche

Vorstand: Walter G. Schües (0), Walther Käding (0).

Chefmathematiker: Karl Todt.

47. Nordstern

Vorstand: Edgar Schnell (V), Dr. Karl Bechstedt (o).

Chefmathematiker: Herbert Eckhardt, Hans Stoebe.

48. Nürnberger

Vorstand: Dr. Hermann Benken (V), Dr. Hans von Eicken (0).

Chefmathematiker: Adolf Goltermann.

49. Pensionsverein

Vorstand: Josef Divora (V), Alfred Ressel (0).

Chefmathematiker: Alfred Ressel.

50. Prima

Vorstand: Alfred Wabnegg (V), Friedrich Eckler (vom Aufsichtsrat delegiert), Fritz Hilpert (st).

Chefmathematiker: Prof. Dr. Georg Nöbeling, Erlangen.

51. Raiffeisendienst

Vorstand: Gottfried Meulenbergh (V), Dr. August Wolf (o),

Reinhold Baurs-Krey (o).

Chefmathematiker: Dr. August Wolf.

52. Rheinisch-Westfälische

Vorstand: Hermann Engemann (o).

Verantwortlicher Mathematiker: Dr. Georg Heubeck

(i. V. Dr. Eberhard Bente).

53. Rothenburger

Vorstand: Otto Garde (V), Dr. Hellmuth Nöbel (st).

Chefmathematiker: Erich Holle.

54. Schweizerische

Vorstand: Generaldirektor: Prof. Dr. Émile Marchand, Direktoren: Dr. Riccardo Jagmetti, Dr. Hans Wyss, Vizedirektoren: Dr. Max Karrer, Josef Meyer, Dr. Ernst Reber, Hans Weber, Charles Zacher.

I. Mathematiker: Dr. Hans Wyss.

Hauptbevollmächtigter für Deutschland: Vizedirektor Albrecht Kressel in München. 
55. Sparkassen-Versicherung

Vorstand: Gustav Ernst (V), Erwin Fix (o).

56. Vereinigte Leben

Vorstand: Berthold Beitz (V), Dr. Wilhelm Hartmann (o),

Dr. Heinrich Rütten (0), Rudolf Meisch (st),

Gunther Albers (st).

Chefmathematiker: Rudolf Meisch.

57. Victoria zu Berlin

Vorstand: Dr. Kurt Hamann (V), Dr. Edmund Haffmanns (st), Wolfgang Knochenhauer (st), Alfred Overhamm (st), Dr. Wolfgang Sachs (st), Wilhelm Seemann (st), Ernst Teckenberg (st).

Chefmathematiker: Dr. Wolfgang Sachs.

57. Victoria am Rhein

Vorstand: Dr. Edmund Haffmanns (o), Alfred Overhamm (0), Dr. Wolfgang Sachs (0), Ernst Teckenberg (0).

Chefmathematiker: Dr. Wolfgang Sachs.

58. Vita

Direktion: Generaldirektor: Dr. W. Linsmayer, Direktor: Dr. E. Röthlisberger, stellv. Direktoren: F. Sauser, C. Stokar, Subdirektoren: P. Brechtbühl, Dr. B. Gabade, L. A. Hürlimann.

Chefmathematiker: Dr. E. Röthlisberger.

Hauptbevollmächtigter für Deutschland: Dr. Herbert . Kaase in Frankfurt a. $M$.

59. Vohk

Vorstand: Gustav Winter (o).

Chefmathematiker: Dr. Josef Siegel.

60. Volkshilfe

Vorstand: Dr. Leo Robert Assmann (o) in Köln, Otto Heinzel (o) in Berlin.

Chefmathematiker: Max Pilz in Berlin.

61. Volkswohl-Bund

Vorstand: Eberhardt Baath (0) in Berlin, Paul Hoffmann (0) in Dortmund.

Chefmathematiker: Dr. Alfred Rosenzweig in Berlin, Gerhard Schalow in Dortmund.

62. Winterthur

Vorstand: Generaldirektor: Dr. Otto E. Pfenninger, Direktor: Roger Breguet.

Chefmathematiker: Roger Breguet.

Hauptbevollmächtigter für Deutschland: Direktor Werner R. Schmitt in München.

63. Witwen- und Waisenkasse

Vorstand: Friedrich Glätzl (V), Dr. Günther Höllring (o).

Chefmathematiker: Albrecht Krupp.

64. Württembergischer Versicherungsverein

Vorstand: Dr. Dr. Max Koppe (o), Dr. Emil Edel (o).

Chefmathematiker: Dr. Dr. Max Koppe.

65. Offentlich-rechtliche Lebensversicherungsanstaiten s. Seite XVI. 


\section{Öffentlich-rechtliche Lebensversicherungsanstalten}

Verband öffentlicher Lebensversicherungsanstaiten

in Deutschland

Verbandsvorsitzender:

Dr. Karl Wilder, Generaldirektor der Anstalt Schleswig-Holstein

Verbandsvorstand:

Dr. Hermann Roth (o), Dr. Walter Domin (o),

Dr. Herbert Dibbert (0)

Verbandsmathematiker: Dr. Herbert Dibbert

1. Hessen-Nassau

Vorstand: Willi Haug (o), Dr. Gerhard Knüfermann (o).

Chefmathematiker: Dr. Gerhard Knüfermann.

2. Rheinprovinz

Vorstand: Dr. Anton Bertgen (V), Dr. Wilhelm Kampmann (0). Chefmathematiker: Walter Kehr.

3. Westfalen

Vorstand: Bernhard Fröhleke (V), Kurt Hellwig (0).

Anstaltsmathematiker: Eberhard Voigt.

4. Schleswig-Holstein

Vorstand: Dr. Karl Wilder.

Mathematiker: Gerhard Köhler.

5. Hannover

Vorstand: Dr. Hans Thunsdorff (V), Dr. Franz Härdrich (o), Hermann Drößler (st).

6. Bayern

Vorstand: Dr. Rudolf Zorn (V), Dr. Michael Daubenmerkl (0), Rupert Zöller (0).

Chefmathematiker: Leopold Baumgartner.

7. Oldenburg

Vorstand: Dr. Erich Muckelmann (V), Dr. Dr. Oskar Schulz (0). Anstaltsmathematiker: Friedrich Humbert.

8. Baden

Vorstand: Alfred Tröbliger (V), Dr. jur. Hans Schleenbecker (0). Mathematiker: Dr. Werner Müller.

9. Braunschweig

Vorstand: Dr. Josef Lammers (o), Stefan Quast (st), Joachim Oestreich (st).

Chefmathematiker: Reinhold Jausel.

10. Sparkassenversicherung

Vorstand: Gustav Ernst (V), Erwin Fix (0).

11. Berlin

Vorstand: Dr. Willy Mäller-Wieland (V), Gerhard Loch (st).

Chefmathematiker: Erich Friede. 